\title{
L'apport de la sociolinguistique à la lexicographie: l'exemple récent des belgicismes dans l'édition du Nouveau Petit Robert $2008^{1}$
}

\author{
Jean-Nicolas De Surmont, Université de Leeds, Leeds, Royaume-Uni \\ (jdesurmont@yahoo.fr)
}

\begin{abstract}
Résumé: Cet article souhaite analyser le contenu de l'inclusion des 48 nouveaux belgicismes dans le Nouveau Petit Robert 2008 (dorénavant NPR 2008). Il retrace les antécédents de collaborations entre différents linguistes et les Éditions Robert pour l'inclusion de belgicismes et évoque le processus qui a conduit à la décision de mettre à jour la nomenclature des belgicismes dans le NPR 2008. Il fait aussi le point sur ces belgicismes en comparant, tableau à l'appui, la présence des belgicismes récemment ajoutés à l'aide d'autres dictionnaires français. À partir de cette comparaison et des données sociolinguistiques fournies dans le cadre du projet de recherche Valibel, il s'interroge enfin sur la pertinence de l'inclusion de ces belgicismes.
\end{abstract}

Mots-clés: FRANÇAIS DE BELGIQUE, METALEXICOGRAPHIE, SOCIOLINGUISTIQUE

\begin{abstract}
The Contribution of Sociolinguistics to Lexicography: The Recent Example of Belgicisms in the Edition of the Nouveau Petit Robert 2008. This article aims to analyse the nature of the 48 new Belgicisms included in the Nouveau Petit Robert 2008 (henceforth NPR 2008). It traces the history of the collaboration between different linguists and the Robert editions regarding the inclusion of Belgicisms and touches on the process leading to the decision to include the list of Belgicisms in the NPR 2008. It also takes stock of these Belgicisms by comparing them with the aid of a table to the Belgicisms recently added by means of other French dictionaries. Lastly, proceeding from this comparison and the sociolinguistic evidence furnished by the research project Valibel, it questions the relevance of the inclusion of these Belgicisms.
\end{abstract}

Keywords: BELGIAN FRENCH, METALEXICOGRAPHY, SOCIOLINGUISTICS

\section{Introduction}

C'est en $1968^{2}$ et non en 1972 comme on l'affirme souvent, que les dictionnaires de langue française ont commencé à inclure des francophonismes. Parmi ceuxci mentionnons les belgicismes qui retiendront notre attention dans ce texte. C'est plus précisément la contribution récente du NPR 2008 dans la mise à jour de ces belgicismes qui sera analysée. L'ajout de 48 belgicismes dans le NPR 2008 à la suite de notre collaboration au projet Valibel s'avère être une contribution historique puisque depuis une vingtaine d'années le Robert n'incluait 
plus de belgicismes. Afin de voir les critères qui ont été retenus pour sélectionner ces belgicismes et la dimension plus ou moins novatrice du choix lexicographique, nous avons dépouillé un corpus de quelques dictionnaires de langue française récents et avons comparé les résultats d'enquêtes en Belgique francophone avec les mots sélectionnés. Au terme de ces comparaisons, différents points de conclusion émergent, permettant d'évaluer les forces et les faiblesses de ces choix.

\section{Antécédents de collaboration des éditions Robert pour l'inclusion des belgicismes}

C'est par l'entremise d'Émile Seutin (Petit Robert (dorénavant PR) en 1983³) et de celle de Maurice Piron ${ }^{4}$ (Grand Robert (dorénavant GR) pour l'édition de 1985) que les Éditions Robert ont ajouté à la nomenclature du PR et du GR de nombreux belgicismes. Le Dictionnaire $d u$ français vivant, l'un des premiers dictionnaires reconnus pour avoir tenu compte du français régional, comptait déjà 270 lexèmes belges en 1973..$^{5}$ Lors d'une conversation en 2003 avec Danièle Morvan, ${ }^{6}$ celle-ci avait mentionné que la nomenclature déjà présente suffisait à ces yeux et une proposition avait été faite de combler les lacunes du GR et du PR.7 Entre septembre 2003 et juin 2004, nous nous sommes attelés à recenser la présence de l'ensemble de la nomenclature des belgicismes du Centre de recherche Valibel (Variétés Linguistiques du français en Belgique ${ }^{8}$ ) dans les dictionnaires suivants: Grand Robert, Banque de données de langue française panfrancophone, dans la base textuelle Frantext et dans le Trésor de la langue française informatisé. Ce travail de longue haleine et minutieux devait pour ainsi dire conduire à l'établissement des choix que Valibel prendrait ensuite sur la pertinence ou non d'inclure certains belgicismes dans le PR permettant aussi la confirmation ou le retrait de plusieurs belgicismes déjà existant (l'enquête sociolinguistique et l'enquête métalexicographique comparée étant complémentaires). Le linguiste ardennais Michel Francard nous enjoignait à noter tout type d'information que ne possédait pas déjà le Centre de recherche Valibel et d'y ajouter ces informations sur une fiche de contrôle. Toute connaissance encyclopédique était évidemment la bienvenue, aidant en même temps à la constitution des données métalinguistiques du Dictionnaire du français en Belgique (dorénavant DFB), projet de recherche en cours au Centre de recherche Valibel de l'Université Catholique de Louvain et qui conduira à la publication d'un dictionnaire (le titre du projet étant celui du dictionnaire à paraître) sur le français de Belgique dont la principale caractéristique novatrice sera la prise en compte de la sociolinguistique par la technique lexicographique.

\section{Le Dictionnaire du français en Belgique}

L'ajout des belgicismes dans le NPR 2008 s'inscrit dans un projet plus vaste, 
celui du Centre de recherche Valibel et plus précisément au sein de la composante lexicographique de ce centre. La composante lexicographique de l'équipe (Régine Wilmet, Geneviève Geron) accueille régulièrement des étudiants et des chercheurs étrangers pour la collaboration au dictionnaire. Or, l'activité de publications métalexicographiques par le centre de recherche nous semble moins importante ${ }^{9}$ que celle émanant de la partie de l'équipe dont les activités sont centrées sur la phonologie du français contemporain (PFC) et sur la sociolinguistique du français en Belgique. Nous avons voulu remédier à cette faiblesse en commentant de façon plus attentive le travail métalexicographique de l'équipe, tout en portant notre attention sur l'ajout des belgicismes dans le NPR 2008. Il convient néanmoins d'évoquer à grands traits les dimensions novatrices qu'apportera le DFB à la lexicographie du français contemporain tant sur le plan méthodologique que sur le plan du contenu.

La majorité des dictionnaires spécialisés sont des encyclopédies (dictionnaires de médecine, d'informatique, de marine) et «beaucoup plus rarement de véritables dictionnaires de langue proprement dit». ${ }^{10}$ Issu de dépouillements importants de corpus linguistiques, de métalinguistique (belges et français surtout), d'enquêtes sociolinguistiques, le DFB, à visée linguistique et encyclopédique, accordera une large part de ses informations au français des médias, écrits et audio-visuels. Le futur DFB semble constituer, selon Marc Van Campenhoudt, un dictionnaire complet. L'ouvrage paraîtra chez De Boeck et suivra vraisemblablement le modèle du dictionnaire de Pierre Rézeau (bilan lexicographique, carte géolinguistique ${ }^{11}$ ), même si de légères différences sont à signaler, comme le fait que l'enquête de vitalité précède l'établissement de la nomenclature et l'adjonction d'images offrant ainsi des développements encyclopédiques importants. Signalons à titre de rappel historique que l'Atlas linguistique de France (1902-1920) d'Edmond Edmont et Jules Gilliéron comportait lui-même 23 points d'enquête en Wallonie; il figure comme précurseur de l'Atlas Linguistique de la Wallonie d'après les enquêtes du dialectologue liégeois Jean Haust, ${ }^{12}$ qui conduit son enquête dans 400 communes, un réseau de points très serrés. L'introduction en lexicographie régionale de cartes géolinguistiques nous semble une grande innovation et témoigne surtout de l'apport théorique et méthodologique de la sociolinguistique sur l'activité lexicographique du français en Belgique. ${ }^{13}$ À ce titre, notre perspective s'est aussi alignée sur celle de Rézeau pour ce qui concerne le dépouillement d'une documentation qui ne s'appuie pas uniquement sur des dictionnaires de référence mais aussi sur des recherches d'attestations provenant de l'Hexagone. ${ }^{14}$ La publication du Dictionnaire des régionalismes de France (dorénavant DRF) sous la direction de Rézeau constitue non seulement une étape dans l'étude du français régional mais aussi l'achèvement d'une entreprise largement inspirée des Matériaux pour l'étude des régionalismes $d u$ français publiés en onze volumes entre 1983 et 2003; elle semble témoigner de la volonté de l'administration française de décentraliser (loi de la décentralisation datant de 1982) ses efforts en vue d'un regain pour les études $\mathrm{du}$ français régional. ${ }^{15}$ 


\section{Les belgicismes du Nouveau Petit Robert 2008}

L'analyse de la nomenclature des belgicismes permet de constater que les belgicismes retenus sont pour la plupart des belgicismes lexicaux; ${ }^{16}$ seul quelques belgicismes sémantiques ont été retenus. Ces particularités lexicales constituent comme beaucoup de topolectismes des emprunts, interférences ou calques du wallon ou du flamand, moins souvent de l'anglais, ainsi que la dérivation suffixale et les changements de sens. ${ }^{17}$ Bien que les topolectismes belges contiennent également des faits syntaxiques, leur description est souvent bien minime dans les dictionnaires et les travaux qui leur sont consacrés sont encore moindres. Parmi ces belgicismes lexicaux certains sont des belgicismes de partage c'est-à-dire, selon De Surmont (2004), des unités lexicales également employées dans d'autres variétés de français que celle de Belgique (Congo, Suisse, Québec). L'extension géographique d'un emploi constitue ce que Pierre Rézeau décrit comme de la dérégionalisation. ${ }^{18}$ La majorité des belgicismes figurent déjà dans le Petit Larousse illustré (dorénavant PLI) et dans le dictionnaire de Georges Lebouc (1998) et cela depuis parfois dix ans ce qui n'est guère étonnant étant donné que le PR n'avait guère ajouté de belgicismes depuis la collaboration du dialectologue liégeois Maurice Piron.

La majorité des belgicismes ont surtout été retenus en fonction des résultats de l'enquête de vitalité sur la base d'un taux de reconnaissance (vitalité passive) supérieure à $90 \%$ parmi les informateurs. ${ }^{19}$ L'enquête de vitalité, comme nous l'avons déjà mentionné, ${ }^{20}$ constitue l'une des particularités du dictionnaire de Valibel en ce qu'elle précède l'établissement de la nomenclature du DFB. À cet égard l'établissement de la vitalité des emplois s'inscrit en partie dans une tradition inaugurée par le Trésor de la langue française (dorénavant TLF), premier dictionnaire ouvert au quantitativisme, même s'il avait négligé les attestations essentiellement littéraires présentant un net décalage avec le français oral. Les enquêtes ont été menées en Wallonie et à Bruxelles tenant compte de l'âge de la scolarité et de la localisation des informateurs et sont plus contrôlées que celle pratiquées par Baetens Beardsmore. Dans le cadre de notre analyse comparative nous avons aussi questionné quelques personnes sur la nomenclature sélectionnée afin de tester les choix opérés par l'équipe de Valibel (Jean-Marie Le Clézio, Jacques De Decker et des étudiants de Bruxelles de provenances variées). Certaines acceptions consignées des belgicismes ont été écartées lorsque le taux de vitalité était estimé trop bas. L'écart entre la forte vitalité d'emploi de certains lexèmes et l'absence de représentation dans les sources métalinguistiques françaises nous semble aussi un facteur récurrent qui a justifié la sélection de certains lexèmes. Il est évident que ce jugement est porté en fonction du dictionnaire français qui sera publié chez De Boeck, ainsi que des acceptions non retenues pour le NPR 2008 étant donné les contraintes d'espace. Cette décision de retenir les belgicismes en fonction des résultats d'une enquête sociolinguistique effectuée sur le territoire francophone de Belgique nous permet d'affirmer que c'est en fonction du lectorat belge du PR qu'ont été retenus les belgicismes et non en fonction des lecteurs français. 


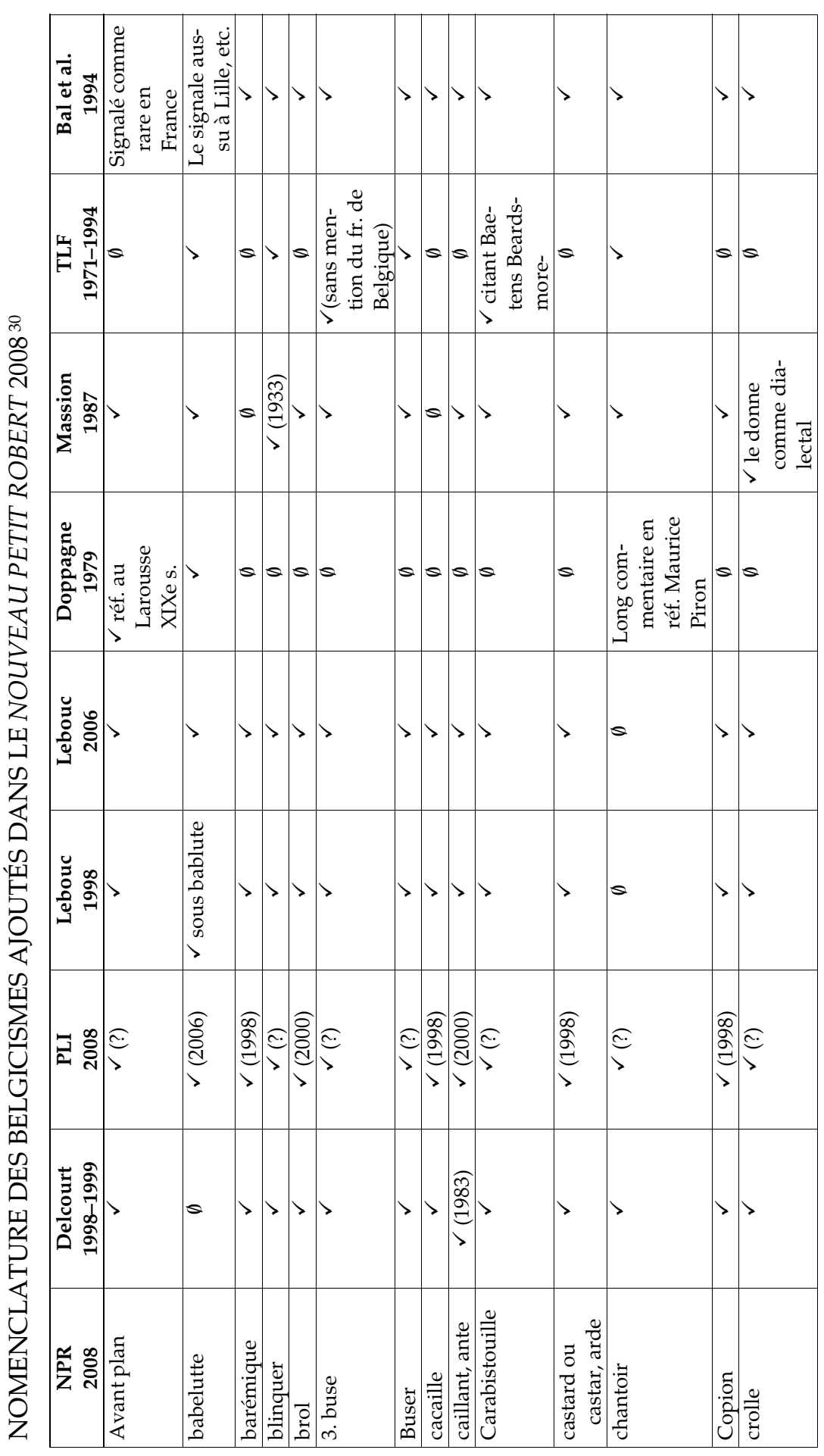




\begin{tabular}{|c|c|c|c|c|c|c|c|c|c|c|c|c|c|}
\hline लं & & & $>>$ & $>$ & $>>$ & $>$ & & $>$ & $>$ & 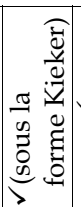 & $>$ & & $>>$ \\
\hline$\frac{\text { मू }}{\frac{1}{7}}$ & & & & & 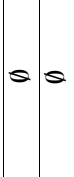 & 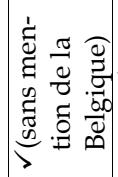 & & & $>$ & $s$ & $>\theta$ & $s$ & $>a$ \\
\hline 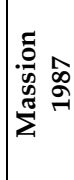 & & & 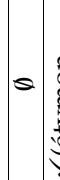 & 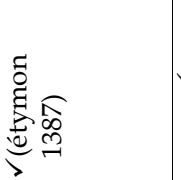 & $>>$ & $>$ & $\otimes>$ & $>$ & $>$ & $>$ & ه & & $>o$ \\
\hline 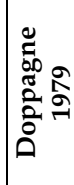 & & & $>$ & 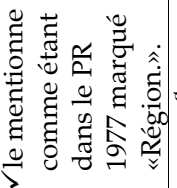 & 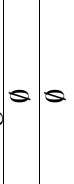 & 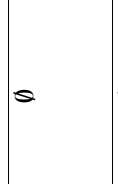 & $s=$ & $s$ & $\theta$ & $\theta$ & $\otimes \theta$ & & \& $s$ \\
\hline 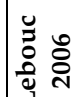 & & $>$ & $>>$ & $>$ & $>>$ & $>$ & $\Delta>$ & $>$ & $>$ & $>$ & $>>$ & & $>>$ \\
\hline 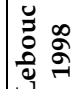 & & $>>$ & $>>$ & $>$ & $>>$ & $>$ & $\Delta>$ & $>$ & $>$ & $>$ & $>>$ & & $>>$ \\
\hline 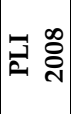 & & 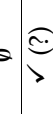 & $\widehat{S}$ & & 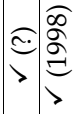 & $\widehat{\Theta}$ & 00 & $=$ & $s$ & 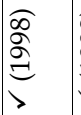 & 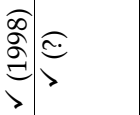 & & $\hat{s}_{s}$ \\
\hline 壱 $\frac{2}{2}$ & & $>$ & $>>$ & $>$ & $>>$ & $>$ & $\Delta>$ & & $>$ & $>$ & $>>$ & & $>a$ \\
\hline 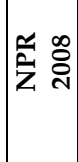 & 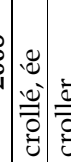 & & 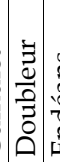 & & 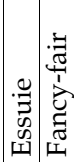 & 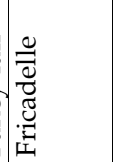 & $\begin{array}{l}: \\
:\end{array}$ & 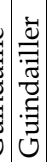 & 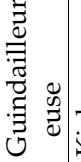 & 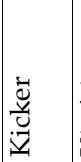 & 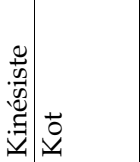 & & 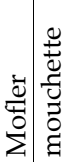 \\
\hline
\end{tabular}




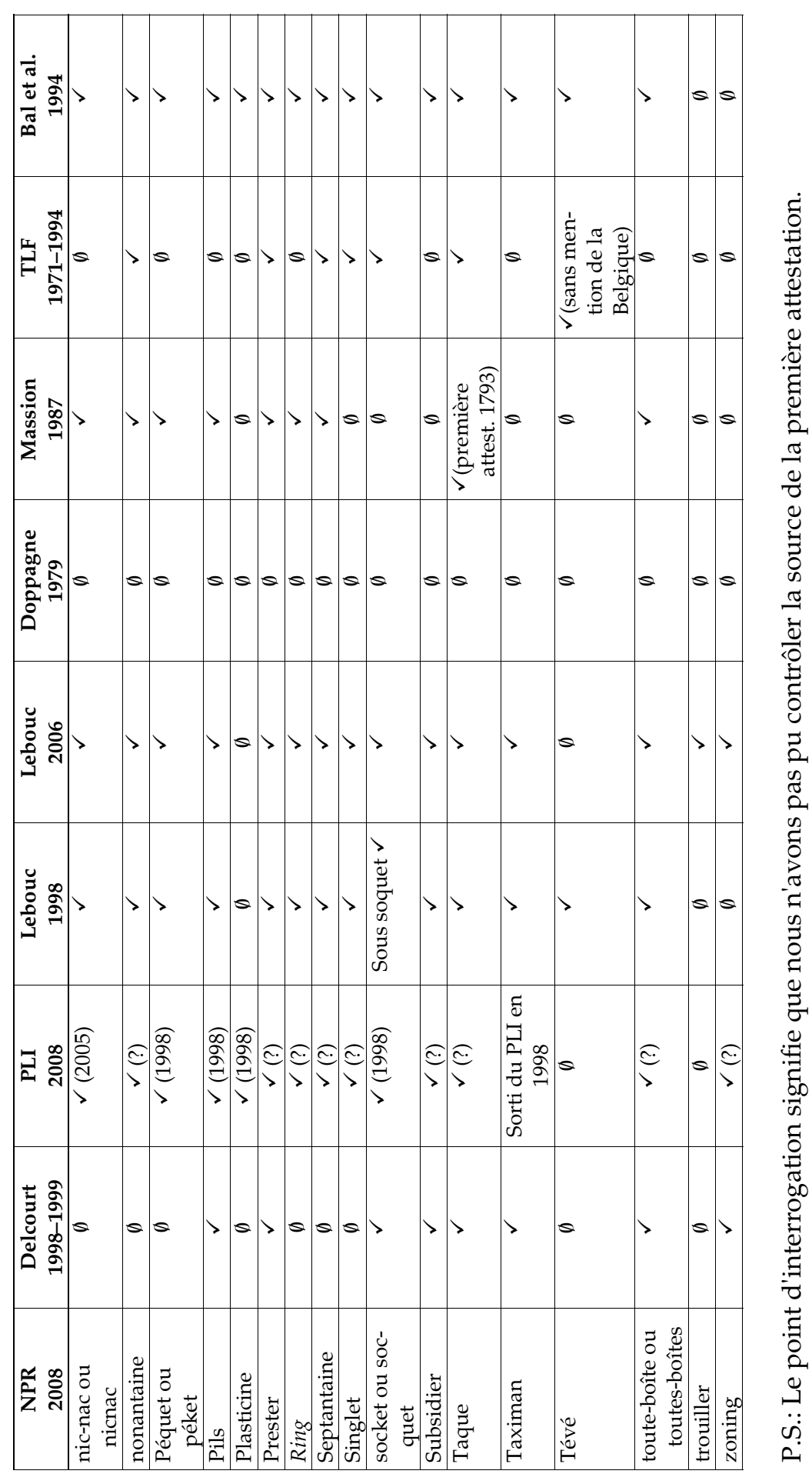


Ces belgicismes, absents du TLF, du Grand Robert de la BDLP ${ }^{21}$ et de la base de données textuelles Frantext et de quelques sources que j'avais contrôlées, se trouvent également dans les corpus des 48 belgicisme du NPR 2008. On peut de même observer, sur la base d'une analyse comparative avec la nomenclature des belgicismes consignée dans le Dictionnaire universel d'Hachette, que les exemples mentionnés, voire les définitions, sont souvent plus courts dans le NPR 2008, même si certains mots apparaissant dans le NPR 2008 ne figurent pas dans le Dictionnaire universel. Les quelques topolectismes de partage avec les helvétismes sont tirés du Dictionnaire suisse romand (dorénavant DSR) d'André Thibault et Pierre Knecht (1997), contrairement au dictionnaire de belgicismes de Christian Delcourt qui consigne des helvétismes sur la base d'autres sources non reprises par l'équipe de Francard, les deux ayant pourtant travaillé en collaboration jadis pour l'élaboration de la base de données textuelles Beltext. ${ }^{22}$

Les améliorations apportées à l'édition 2008 sont évidentes quant à l'harmonisation du marquage diatopique qui a tenu compte de nos propositions émises lors du congrès EURALEX de 2004 (Voir De Surmont 2004). Nous avions effectivement remarqué que le GR, comme de nombreuses sources lexicographiques, souffrait d'un manque d'homogénéité des marques diatopiques. Ainsi, l'ensemble des belgicismes est marqué «Région. (Belgique)». C'est là, il nous semble, vouloir faire preuve d'une plus grande clarté dans la présentation des informations métalinguistiques de l'article lexicographique. Notons que l'information étymologique consignée de plusieurs des belgicismes comporte des données intraterritoriales (flamand, d'origine wallonne), tout autant qu'extraterritorial (anglais) ou d'origine incertaine. Étant donné la nature des enquêtes effectuées, nous pensons que les belgicismes d'origine wallonne comme d'origine dialectale sont le résultat à la fois de la redéfinition du dialecte ${ }^{23}$ comme pratique patoisante liée à la disparition même des pratiques patoisantes, en même temps que des échanges féconds entre les sociétés traditionnelles et modernes et la mobilité des locuteurs. Le nombre de locuteurs patoisant diminue ce qui n'empêche évidemment pas le fonds dialectal d'alimenter le français régional. Il est intéressant de signaler à cet effet que l'origine wallonne ou flamande relevé par le GR3 n'est pas toujours consignée dans le NPR 2008. C'est le cas de buse /échec à un examen/ dont le sens étymologique wallon est /haut-de-forme/, de crolle /boucle de cheveux/.24 Dans le cas de cumulet, si le GR3 affirme qu'il s'agit d'un «mot wallon d'orig. obscure», la première syllabe représente probablement $c u l$, le NPR 2008 affirme quant à lui qu'il est issu du moyen néerlandais tumulen /se tourner avec/, peut-être, sous l'influence du français cul (Picardie; Belgique). Ceci confirme la position de Jacques Pohl, dont l'ouvrage, paru en 1962, fait office de premier livre "purement descriptif» pour le français de Belgique. ${ }^{25}$ Le mot endéans fait l'objet d'une marque diatopique associée à une marque diachronique régressive dans le GR3 (vx ou régional (Belgique)), et didact. (droit, admin.) à une information étymologique exhaustive dans le NPR 2008: fin XIV (Tournai) de 1 en, 1 de et de l'ancien français enz «dans», du latin intus /à l'intérieur/. Un réel travail a été fait au niveau du 
contrôle des sources et de l'information étymologique en s'inspirant des données des rubriques «Histoire et Bilan métalexicographique» qui apparaîtront dans le DFB permettant d'améliorer ou de préciser l'information étymologique voire de créer la dispersion topolectale des emplois. ${ }^{26}$ Les bilans métalexicographiques de François Massion ont été particulièrement utilisés sur le plan diachronique, ce qui est en l'occurrence le cas de endéans où les auteurs ont préféré indiquer «fin XIV, 1387» comme l'avait fait François Massion en 1987.27 Pour les attestations plus récentes et la dispersion géographique, notamment en Afrique, le dictionnaire de Christian Delcourt est particulièrement significatif, bien qu'il a été peu exploité par l'équipe Valibel si l'on en juge le fait que les topolectismes de partage ne sont pas mentionnés. Certains traitements nous semblent néanmoins étonnants, comme guindaille /sortie joyeuse et bien arrosée/ dont le NPR 2008 fait remonter la première attestation à 1889. Il s'agit probablement d'une altération de godaille / ripaille/ qui remonterait au moyen néerlandais goed ale, mot à mot «bonne (goed) bière (ale)». Or le GR3, dont la publication est pourtant antérieure, antidate la première attestation de 9 ans: «mot wallon, p.-ê. de godaille d'après guinse «beuverie», du francique winst / profit/, ou d'après 2. guindal». C'est aussi le cas de mofler dont le GR3 affirme qu'il provient du wallon (liégeois) mofe /moufle, gros gants/; d'où «frapper d'un coup au visage» alors que le NPR 2008 affirme mofler: 1930 est un mot d'origine incertaine. Le mot taque apparaît comme non marqué dans le PLI et dans le GR3 ce qui peut laisser soupçonner la nécessité de le marquer dans le NPR 2008. On constate aussi une extension topologique de la zone d'emploi du mot taximan qui passe d'un emploi marqué français d'Afrique dans le GR3 à un topolectisme aussi bien employé en Afrique qu'à Madagascar et en Belgique dans le NPR 2008. ${ }^{28}$ Le mot Tévé présente une autre particularité. Dans le GR3 aucune marque diachronique régressive apparaît pour l'emploi en français de France alors que le NPR 2008 le mentionne avec la marque Belgique. Une divergence de datation apparaît aussi pour zoning où le GR3 mentionne 1934 alors que le NPR 2008 mentionne 1994. Le GR3 ne mentionne par ailleurs aucune restriction géographique d'emploi alors que le NPR 2008 le prétend être un belgicisme. ${ }^{29}$ Bien qu'ici le bilan métalexicographique soit mitigé c'est surtout la divergence de résultat entre le GR3 et le NPR 2008 qui nous semble douteux. Une antidatation nous semble tout à fait normale mais de post-dater un emploi de 60 ans d'un produit des dictionnaires Robert antérieur à un autre nous paraît étonnant.

\section{Conclusion}

Il faut constater que la représentation de la Belgique dans les dictionnaires Robert a laissé à désirer depuis une vingtaine d'années. On remarque en outre que, contrairement au français québécois, le français de Belgique suscite moins d'intérêt que n'en a suscité en tout les cas la dialectologie wallonne. La publication de dictionnaires universitaires du français de Belgique ne suscite pas non plus un intérêt politique comme c'est le cas au Québec, cela étant peut-être dû 
au fait qu'au Québec le désir d'émancipation socio-économique des élites canadiennes françaises et la libération culturelle qui s'en est suivie, ont toujours été intimement liés aux questions linguistiques et s'inscrit dans une politique bilatérale mise en place dans les années 1960.

Les problèmes communautaires, caractéristiques pour la Belgique sont nuisibles pour l'unité belge et l'on ne saurait nier le fait que la Belgique (pays de réformes, de chantiers et de compromis ...) viennent derrière la Suisse et le Québec dans l'élaboration d'un dictionnaire des particularités lexicales incluant des informations de natures historique, philologique et métalinguistiques, comme le Dictionnaire historique du français québécois (initié par Marcel Juneau et continué par Claude Poirier) et le DSR et le DRF.

Les ajouts de belgicismes dans le NPR 2008 veulent être plus représentatifs de la vitalité des usages sur la base des acquis des enquêtes sociolinguistiques. Les résultats de ces enquêtes ont donné lieu à la constitution de corpus importants et servent en quelque sorte à mieux contrôler la dispersion géographique et sociolinguistique des usages. Cela permet de confirmer la pertinence des propositions d'Emile Seutin dont les propositions se faisaient sur la base des ouvrages passés et d'intuitions personnelles, et non pas forcément acceptées par les éditions Robert. La question qui se pose dorénavant n'est plus la question de la validité des intuitions personnelles de linguistes mais la représentation des ressources textuelles qui sont à la disposition des linguistes de l'école néolouvaniste de sociolinguistique. L'échantillon de ressources textuelles est-il représentatif des différentes productions orales observables sur le territoire de la Communautés Wallonie-Bruxelles? ${ }^{31}$ Tout au moins ce renouvellement de la technique lexicographique sur la base des acquis de la sociolinguistique tend à vouloir s'écarter de la tradition puriste qui procédait par le couple dites/ne dites pas et donnait une vision morcelée de la langue. Néanmoins la composante géolinguistique, en particulier en situation de contact et en tenant compte de l'évolution historique des contacts entre Flamands et francophones devrait aussi orienter la rubrique historique. ${ }^{32}$

Dans la mesure où la nomenclature des ajouts de belgicismes du NPR 2008 préfigurant au $\mathrm{DFB}$, ou tout au moins répondant au cadre méthodologique du groupe de recherche Valibel, constituent selon nous un élément objectivable de réflexion sur les méthodes lexicographiques en cours actuellement en Belgique francophone. En outre cela nous aura permis de mieux connaître les pratiques récentes de propositions topolectales aux dictionnaires de langue générale du français.

À la lumière de ces nouvelles réflexions il serait intéressant dorénavant de mieux cerner le vaste domaine des topolectismes de partage en relativisant les frontières érigées en barrières entre le français de référence et le français régional. En outre la question des mots attestés en Belgique avant de l'être en France et passés dans l'usage courant et les naissances indépendantes ${ }^{33}$ devraient nous conduire à une réflexion plus globale sur la définition du belgicisme prenant en compte non seulement les questions géolinguistiques comme avant mais aussi les questions sociolinguistiques. 


\section{Notes}

1. Nos remerciements vont à Michel Francard d'avoir mis à notre disposition le matériel de l'enquête géolinguistique et surtout Geneviève Géron de nous avoir assisté régulièrement dans la conduite de nos recherches.

2. C'est en effet le PLI sorti à l'été 1967, dont la sortie coïncide avec le passage du général De Gaulle au Québec, qui consigne quelques québécismes (bleuet, coureur des bois, débarbouillette, millage, poudrerie, tuque et verge) et non le Dictionnaire du français vivant (1972) comme on a l'habitude de le mentionner. Cette même édition du PLI voit disparaître la formule calquée sur celle de Voltaire: «un dictionnaire sans exemples est un squelette», exemple ayant été substitué à citation.

3. Conversation personnelle Emile Seutin, le 30 octobre 2007. Parti de la Belgique vers le Québec en 1963, Seutin devient professeur de l'Université de Montréal. Il proposa 180 belgicismes dans le PR. Environ 80 pour la première fois en 1984 et ensuite progressivement pendant une dizaine d'années à Josette Rey Debove. Piron et Seutin ne se sont jamais concertés dans leur travail ce qui fait que le PR et le GR pouvant être très divergeant dans leur nomenclature des belgicismes.

4. Notons que Piron a aussi collaboré au Grand Larousse encyclopédique et au Trésor de la langue française.

5. Voir Jacques Pohl (1979: 136). Il ne comptait pourtant alors que 230 québécismes alors que dans le PLI la présence de québécisme a toujours été plus importante que celles des francophonismes des auteurs communautés de langue française. Marion Spickenbom (1996) mentionne qu'en 1953, Maurice Piron évalue à 150 le nombre de belgicismes consignés dans les dictionnaires généraux de langue française, chiffre porté à 240 vingt ans plus tard avec une tendance à ne considérer alors que les belgicismes lexicaux. En 1972, le Dictionnaire du français vivant de Bordas répertorie 270 belgicismes et en 1973, 292. La liste ira en augmentant, Albert Doppagne en recense 405, Heinz Jürgen Wolf 500.

6. Conversation personnelle, octobre 2003.

7. Conversation personnelle, mars 2007. Mentionnons, qu'en août 2006, le site Internet de Valibel indiquait que l'édition 2007 du PR allait ajouter à sa nomenclature des belgicismes ou laissait sous-entendre déjà cela alors que ce n'est en fait que fin juin 2007 à la sortie de l'édition 2008 que les nouveaux belgicismes ont été ajoutés.

8. Localisé à l'Université Catholique de Louvain, ce projet de recherche constitue l'un des maillons importants de la mise sur pied par Bernard Quemada d'un trésor des variétés du français panfrancophone et présente à ce titre des liens importants avec le réseau de Claude Poirier.

9. On notera cependant une partie des travaux issus de notre séjour à Valibel: De Surmont $(2004,2005)$ et à paraître et les contributions de Michel Francard, Geneviève Geron et Régine Wilmet plutôt axée sur l'apport sociolinguistique à la métalexicographie qu'à la métalexicographie comme telle.

10. Marc Van Campenhoudt (2000: 127).

11. Les cartes géolinguistiques présentent les zones d'ombrage en dégradé variant, au dixième près, selon le taux de reconnaissance pour tout le territoire francophone (Wallonie et Bruxelles incluant aussi Comines) et ont pour certaines déjà été établies entre 1999 et 2002 (cela a été systématiquement utilisé par Pierre Rézeau (en 2001) pour la première fois). (Nous remer- 
cions Geneviève Geron de nous avoir «gracieusement» fait part la carte de aboutonner.) D'autres cartes sont parues dans les contributions collectives de Francard, Geron et Wilmet. Ajoutons que la rubrique "Citation» comprend des exemples extraits de la toile et d'émissions de radio ce qui constitue en lexicographie régionale de langue française un élément très novateur. En outre, la rubrique «Emploi» confronte l'oral et l'écrit et distribue les taux de reconnaissance et d'emploi des lexèmes sur le territoire francophone de la Belgique. À ce jour, la rubrique historique recense essentiellement des sources métalinguistiques et la forme de ces rubriques rappelle celle instituée par le TLF. Le fond dialectal bien que connu par certains membres de l'équipe (Geneviève Geron, Régine Wilmet, Michel Francard) n'est pas repris systématiquement dans la rubrique historique comme le faisait François Massion (1987) de manière détaillée. Bien que peu mise en évidence, la dimension géolinguistique des Chasses aux Belgicismes de Bal et al. a été pris en compte puisque chacun des co-auteurs enquêtait dans sa région: Albert Doppagne couvrant la région de Huy et de Bruxelles, Joseph Hanse celle de Florenne, Bal celle de Charleroi, André Goosse les Ardennes notamment près de Laroche, L. Warnant couvrait la région de Liège et madame Lenoble-Pinson celle de Libramont. Conversation personnelle Michèle Lenoble-Pison, 29 novembre 2007.

12. Mentionnons que Francard (1995-1996) s'est intéressé à la technique lexicographique du Dictionnaire liégeois de Jean Haust. L'ensemble de sa production antérieure nous laisse penser qu'il devrait être plutôt considéré comme héritier de la dialectologie wallonne que de la lexicographie française et qu'il marque à sa manière la transition de l'hégémonie de l'école dialectologie wallonne vers la prise en compte du français de Belgique, trop longtemps considéré que par les puristes. L'enquête de l'ALF cartographiait surtout la variation phonétique au détriment de la variation sociolinguistique. Plusieurs facteurs ont entraîné la définition de nouveaux projets intégrant une composante sociolinguistique variationniste: «l'évolution économique et sociale qui a réduit l'importance démographique des zones rurales aux dépens des zones urbaines, la mise en chantier d'atlas dans les domaines nouveaux à la structuration linguistique particulière comme ceux de la 'Romania Nova', la constitution de la sociolinguistique comme discipline [...]» (Jean-Paul Chauveau 2003: 78). Voir aussi JeanPaul Chauveau (1995-1995) sur l'entreprise de Jean Haust, et Guylaine Brun-Trigaud, Yves Le Berre et Jean Le Dû (2005) sur l'ALF.

13. On consultera à ce propos le site du réseau EFF (Étude du français en francophonie) à l'adresse suivante: http://www.eff.auf.org/article.php3?id_article=25 et Michel Francard, Geneviève Geron et Régine Wilmet (2003: 140). Comme le soulignent avec raison ces mêmes auteurs (p. 141), l'apport de cette discipline à la constitution de la nomenclature reste très limité, malgré les intentions déclarées dans la plupart des ouvrages déjà publiés en langue française. Notons néanmoins les travaux fondateurs de Heinz Fuchs.

14. Voir Francard, Geron et Wilmet (2003: 146) et De Surmont (2004). Notre travail initial à Valibel consistait notamment à rechercher des attestations que les autres membres de l'équipe n'avaient pas trouvées en s'appuyant sur les pages Internet récentes, sur la base Frantext et sur des recherches à partir du moteur de recherche Google.

15. Le dictionnaire écrit en collaboration avec Pierre Rézeau a reçu une bonne critique de toute part, ses mérites ont aussi été vantés par A. Goosse (2007: 87). Voir Pierre Rézeau (2007: 7).

16. La lexicographie variationniste dispose à cet égard de plus d'outils que la grammaire. Ainsi dans l'édition 2007 du Bon usage, le grammairien André Goosse peut s'appuyer sur un corpus linguistique fort important pour la mise à jour de sa grammaire mais en revanche le corpus 
métalinguistique d'articles, de dictionnaires ou d'études portant sur la syntaxe du français régional a fait l'objet de très peu de travaux. C'est donc à la lexicographie qui décrit parfois des faits syntaxique que la grammaire de référence s'en remet encore en 2007. André Goosse s'est ainsi approvisionné aux Dictionnaires des régionalismes de France, dictionnaire que nous n'avions pas dépouillé en 2004 et qui en toute logique ne semble pas avoir été pris en compte dans les belgicismes ajoutés à l'édition 2008 du PR, puisqu'aucun topolectisme de partage figurant dans le NPR 2008 ne figure dans le DRF dirigé par Pierre Rézeau alors que celui-ci fait pourtant référence régulièrement au français de Belgique.

17. La classification des québécismes par Claude Poirier (1995) a été adoptée à plusieurs reprises par Michel Francard par la suite, bien que sur le plan méthodologique Francard a pris ses distances vis-à-vis du point de vue restrictif de Poirier. Dans le cas du néerlandais il est permis de se demander comme A. Goose qui du français ou du néerlandais est le receveur ou le donneur. (Voir Goosse 2007: 85.)

18. 2003: 131. Le problème de la régionalisation et de la dispersion géographique des emplois et de la dérégionalisation apportent à réfléchir sur la variation linguistique francophone en Afrique noire. Ainsi Claude Frey propose le terme de «sous-région» pour désigner l'ensemble géographique homogène comprenant plusieurs états africains (pour le concept de «sousrégion» voir Klinkenberg 2008: 85). Voir Claude Frey (2003: 167), voir aussi Piron (1973: 295304). Piron considère comme belgicismes les particularités communes à plusieurs provinces de Belgique.

19. Sur les 48 belgicismes consignés, seuls 2 d'entre eux nous semblent poser un problème, comme chantoir qui n'est pas consigné dans la majorité des sources métalinguistiques consultées et inconnu des personnes que nous avons consultées y compris Jacques De Decker, ainsi que et des informateurs de Francard lui-même. Il est fort probable que Francard ait subit l'influence de Maurice Piron malgré les données de l'enquête et des sources métalinguistiques (voir à cet égard le commentaire du lexicographe ardennais et de ses deux collaboratrices 2003: 144-145). Le taux de reconnaissance de mofler est moins élevé que les autres lexèmes malgré sa continuelle présence dans les sources métalinguistiques belges y compris dans le GR3. L'enquête de Valibel s'appuie sur un questionnaire écrit adressé à 86 informateurs en plus d'une banque de données textuelles orales importantes obtenues à partir d'enregistrements réalisées avec quelques 530 francophones wallons et bruxellois. (Voir Francard, Geron et Wilmet 2002a: 21; 2003: 142.) Notons que la vitalité selon l'âge, le sexe ou le degré de scolarité est mélangée aux variables régionales et la vitalité selon l'origine géographique est également mélangée aux autres variables mentionnées. L'ensemble de ces variables est corrélée avec les différences de réalisations linguistiques au sein du même système linguistique.

20. Voir De Surmont (À paraître).

21. Mentionnons que même si la BDLP (Base de données lexicographiques panfrancophone) ne serait que peu employée pour la nomenclature de Valibel en novembre 2003, la nomenclature du Delfrabel (Dictionnaire électronique du français de Belgique) comportait 5124 entrées.

22. Source: Conversation personnelle Geneviève Geron, août 2003.

23. Dans le volume de l'Encyclopédie de la Pléiade consacré au langage dirigé par André Martinet, Jean Fourquet (cité par Jean Séguy 1973: 65) prétend «que c'est l'expérience de diversités à l'intérieur de ce qu'on considère comme une langue, et les dénominations de choses n'étant pas identiques, ont des ressemblances évidentes, et que, de façon générale, les différences observées ne sont pas telles qu'elles empêchent la compréhension». En 1978 Patrick Charau- 
deau (1978: 16) écrit que le dialecte est considéré comme une variété régionale d'une langue. Or, selon la définition plus récente de Marina Yaguello (1988: 154), le mot dialecte possède un sens linguistique et un sens sociolinguistique. Au sens linguistique il signifie la variété d'une langue et au sens sociolinguistique le parler vernaculaire non normé et le plus souvent non écrit n'ayant pas le statut de langue. On pourra aussi se reporter à Léon Warnant (1973: 100).

24. Même si dans ce cas la vitalité de l'emploi à diminué étant donné la disparition progressive du référent, il aurait pu être intéressant de mentionner l'origine wallonne du mot puisque le sens /haut de forme/ est une acception connue du mot en français de Belgique. Plus encore le lien entre le chapeau et l'échec aux examens aurait pu être évoqué.

25. 1962: 17. Voir A. Goosse (2007: 87).

26. On notera que l'apport de la sociolinguistique, peut-être mieux documenté pour la dialectologie wallonne, l'est encore fort peu pour le français de Belgique. Voir Albert Doppagne (1978: 50) pour une critique sur la dispersion du français régional. Voir aussi Michel Francard, Geneviève Geron et Régine Wilmet $(2002: 13,14)$.

27. Notons que la recherche du DFB ne s'inscrit pas dans une perspective diachronique ce qui peut expliquer les lacunes sur ce plan (Voir Francard, Geron et Wilmet 2002a: 15).

28. Il aurait été pertinent ici de préciser la zone géographique entendue par la marque topolectale «Afrique», sachant que la zone qu'elle couvre est plutôt vaste, témoignant peut-être d'une vision fédéraliste de l'Afrique. Voir Claude Frey (2003: 173). Ici la marque topolectacle diffère en quelque sorte d'une marque synthétique alors que pour le français régional d'Europe, le caractère régional est beaucoup plus limité géographiquement.

29. La question de la dispersion des emplois a été discutée par De Surmont (à paraître) et Cleante (2000: 9-10).

30. Nous n'avons pas retenu Albert Doppagne (1978) puisque sa nomenclature est plus réduite. La date figurant à côté du PLI est la date d'entrée dans le dictionnaire lorsqu'elle nous est connue.

31. Voir Michel Francard, Geneviève Geron et Régine Wilmet (2002b: 74).

32. On consultera ici avec profit l'ouvrage de Joep Kruijsen (1995) sur les emprunts romans dans les dialectaux limbourgeois de la Hesbaye qui montre qu'avant 1962 on a un maintien des éléments français empruntés avant la loi de 1962.

33. Voir André Goosse 2007: 97.

\section{Bibliographie}

Baetens Beardsmore, Hugo. 1971. Le français régional de Bruxelles. Bruxelles: Presses Universitaires de Bruxelles.

Brun-Trigaud, Guylaine, Yves Le Berre et Jean Le Dû. 2005. Lectures de l'Atlas linguistique de la France de Gillérion et Edmont, Du temps dans l'espace. Paris: Éditions du Comité des travaux historiques et scientifiques.

Charaudeau, P. 1978. Les conditions linguistiques d'une analyse du discours. Thèse soutenue à l'Université Charles-de-Gaulle Lille III.

Chauveau, Jean-Paul. 2003. Histoire des langues romanes et géographie linguistique, Romanische Sprachgeschichte und Sprachgeographie. Ernst, Gerhard, Martin-Dietrich Glessgen, Christina Schmitt et Wolfgang Schweickard (Éds.). 2003. Romanische Sprachgeschichte. Ein Internatio- 
nales Handbuch zur Geschichte der romanischen Sprachen/Histoire linguistique de la Romania. Manuel international d'histoire linguistique de la Romania. Tome 1: 72-90. Berlin/New York: Walter de Gruyter.

De Surmont, Jean-Nicolas. 2004. Caractéristiques et variations du marquage diatopique. Williams, Geoffrey et Sandra Vessier (Éds.). 2004. Proceedings of the Eleventh Euralex International Congress, Euralex 2004, Lorient July 6-10, 2004: 779-786. Lorient: Université de Bretagne Sud.

De Surmont, Jean-Nicolas. 2005. Quelques remarques sur les belgicismes métalinguistiques. Présence francophone 65: [198]-211.

De Surmont, Jean-Nicolas. À paraître. Contourner la marque diatopique: vitalité des emplois et présence des topolectismes dans les dictionnaires de variétés de français. Actes du colloque de Chypre 2006, sur la marque lexicographique.

Francard, Michel. 1995-1996. La technique lexicographique du Dictionnaire liégeois. Les dialectes de Wallonie, t/M 23-24: 221-239.

Francard, Michel, Geneviève Geron et Régine Wilmet. 2002a. Diffusion et vitalité des particularités lexicales du français en Belgique: une enquête sociolinguistique. Wakely, Richard (Éd.). 2002. Les Belges: enregistreurs de tous les usages. Actes d'un colloque qui s'est tenu à l'Université d'Édimbourg les 7 et 8 avril 2000 et publiés en bénéficiant de l'Ambassade de France en Grande Bretagne: 11-32. Édimbourg: Université d'Édimbourg.

Francard, Michel, Geneviève Geron et Régine Wilmet. 2002b. La banque de données VALIBEL: des ressources textuelles orales pour l'étude du français et à Bruxelles. Pusch, Claus D. et Wolfgang Raible (Éd.). 2002. Romanistische Korpuslinguistik. Korpora und gesprochene Sprache/Romance Corpus Linguistics. Corpora and Spoken Language: 71-80. Tübingen: Gunter Narr.

Francard, Michel, Geneviève Geron et Régine Wilmet. 2003. Les 'belgicismes' sont-ils 'belges'? La nomenclature du Dictionnaire du français en Belgique. Nobel, Pierre (sous la dir. de). 2003. Variations linguistiques Koinè, dialectes, français régionaux: 137-150. Besançon: Presses Universitaires de Franche-Comté.

Frey, Claude. 2003. Identités lexicales et variétés de français en France et hors de France: tendance centripètes et centrifuges des formes et des cultures. Nobel, Pierre (sous la dir. de). 2003. Variations linguistiques Koinè, dialectes, français régionaux: 165-190. Besançon: Presses Universitaires de Franche-Comté.

Goosse, André. 2007. Belgicismes exportés. Cahiers de lexicologie 91(2): 85-102.

Klinkenberg, Jean-Marie. 2008. À propos du traitement lexicographique des belgicismes. Bavoux, Claudine (Éd.). 2008. Le français des dictionnaires: L'autre versant de la lexicographie française: 7888. Bruxelles: De Boeck.

Kruijsen, Joep. 1995. Geografische patronen in taalcontact, Romaans leengoed in de Limburgse dialecten van Haspengouw. Amsterdam: P. J. Meertens-Instituut.

Piron, Maurice. 1973. Les belgicismes lexicaux: essai d'un inventaire. Mélanges Paul Imbs: 295-304. Strasbourg.

Pohl, Jacques. 1962. Témoignages sur la syntaxe du verbe dans quelques parlers français de Belgique. Bruxelles: Palais des Académies.

Pohl, Jacques. 1979. Les variétés régionales du français, études belges (1945-1977). Bruxelles: Éditions de l'Université de Bruxelles.

Poirier, Claude. 1995. Les variantes topolectacles du français. Francard, Michel et Daniel Latin (Éds.). 1995. Le régionalisme lexical: 13-56. Louvain-la-Neuve: De Boeck, AUPELF-UREF. 
Rézeau, Pierre. 2003. De la cancoillotte à la tartiflette (et à la boîte chaude) avec le Dictionnaire des régionalismes de France. Nobel, Pierre (sous la dir. de). 2003. Variations linguistiques Koinè, dialectes, français régionaux: 128-136. Besançon: Presses Universitaires de Franche-Comté.

Rézeau, Pierre. 2007. Introduction. Rézeau, Pierre (études rassemblées par). 2007. Richesses $d u$ français; Géographie linguistique. Vol. 1. Bruxelles: De Boeck.

Séguy, Jean. 1973. Les Atlas linguistiques de la France par régions. Langue française 18: 65-90.

Spickenbom, Marion. 1996. Belgizismen in französischen Wörterbüchern und Enzyklopädien seit Anfang dieses Jahrhunderts. Münstersche Beiträge zur Romanischen Philologie 16. Münster: Nodus.

Van Campenhoudt, Marc. 2000. De la lexicographie spécialisée à la terminographie: vers un métadictionnaire? Thoiron, Philippe et Henri Béjoint. 2000. Le sens en terminologie: 127-152. Travaux du CRTT. Lyon: Presses Universitaires de Lyon.

Warnant, Léon. 1973. Dialectes du français et français régionaux. Langue française 18: 100-125.

Wolf, Heinz Jürgen. 1991. Französische Sprachgeschichte. Heidelberg/Wiesbaden: Quelle \& Meyer. Yaguello, Marina. 1988. Catalogue des idées reçues sur la langue. Paris: Éditions du Seuil.

\section{Corpus métalinguistique}

Bal, Willy, Albert Doppagne, André Goosse, Joseph Hanse, Michèle Lenoble-Pinson, Jacques Pohl et Léon Warnant. 1994. Belgicismes, inventaire des particularités lexicales du français de Belgique. Louvain-la-Neuve: Éditions Duculot.

Cleante [pseud. de Louis Chalon]. 2000. Tours et expressions de Belgique. Prononciation, grammaire, vocabulaire. Bruxelles: Duculot.

Davau, Maurice, Marcel Cohen et Maurice Lallemand. 1973. Dictionnaire du français vivant. Londres: Harrap.

Delcourt, Christian. 1998-1999. Dictionnaire du français de Belgique. 2 vol. Bruxelles: Éditions Le Cri.

Doppagne, Albert. 1979. Belgicismes de bon aloi. Bruxelles: Fondation Charles Plisnier, Office du Bon langage.

[EN COLL.]. Dictionnaire universel. Paris: Hachette.

[EN COLL.]. Petit Larousse illustré. Paris: Larousse. [Différentes éditions consultées]

Fuchs, Heinz. 1988. Untersuchungen zu Belgizismen, Zu Ursprung und Verbreitung lexikalischer Besonderheiten des belgischen Französisch. Bonner Romanistische Arbeiten 29. Frankfurt/Bern/New York/Paris: Peter Lang.

Hanse, Joseph. 1971. Chasse aux belgicismes. Bruxelles: Fondation Charles Plisnier.

Knecht, Pierre (sous la direction de) conçu et dirigé par André Thibault, avec la collaboration de Gisèle Boeri et Simone Quenet. 1997. Dictionnaire suisse romand. Particularités lexicales $d u$ français contemporain. Carouge/Genève: Éditions Zoé.

Lebouc, Georges. 1998. Le Belge dans tous ses états. Dictionnaire de belgicismes, grammaire et prononciation. Paris: Éditions Bonneton.

Lebouc, Georges. 2006. Dictionnaire de belgicismes. Bruxelles: Éditions Racine.

Massion, François. 1987. Dictionnaire de belgicismes. Frankfurt am Main/Bern/New York/Paris: Peter Lang.

Rézeau, Pierre. 2001. Dictionnaire des régionalismes de France. Géographie et histoire d'un patrimoine linguistique. Bruxelles: De Boeck \& Larcier/Éditions Duculot. 
Trésor de la langue française. Dictionnaire de la langue du XIX et du XXe siècle (1789-1960). 1971-1994. 16 vol. Paris: Éditions du Centre national de la Recherche Scientifique (vol. 1-10)/Gallimard (vol. 11-16).

\section{Informateurs}

Jean Marie Le Clezio, 17 octobre 2007.

Jacques De Decker, 17 octobre 2007. 